fe tätig. Das macht 10\% ihrer gesamten Tätigkeiten aus. Die Pflegekraft nimmt Aufträge dann an, wenn sie Kapazitäten frei hat. Ihre Arbeitszeit und die jeweilige Gestaltung der Tätigkeiten bestimmt sie im Wesentlichen selbst. Sie unterhält für ihre selbstständige Tätigkeit ein eigenes Arbeitsbüro.

Das Gericht hat den Status als Selbstständige bestätigt. Die Altenpflegerin war im Durchschnitt lediglich circa einmal pro Woche und damit nur gelegentlich bei einem der externen Unternehmen tätig gewesen. Beginn und Ende der Tätigkeit standen vorab fest. Anders als die festangestellten Kräfte hat sich die Tätigkeit der Altenpflegerin auf die reinen Nachtwachentätigkeiten beschränkt, die sie eigenverantwortlich ohne Weisungen und ohne weiteres Eingebunden- sein in den Betriebsablauf verrichten konnte. Die festangestellten Pflegekräfte hatten während der Nachtschicht nicht nur die Nachtwache zu halten, sondern weitere Tätigkeiten zu verrichten, wie etwa Dinge für den folgenden Tag vorzubereiten oder Arbeiten zu erledigen, die liegen geblieben waren. Die Altenpflegerin hat nicht an Dienstbesprechungen oder Ähnlichem teilgenommen und war dazu auch nicht verpflichtet.

Ein weiterer Fall: Eine (examinierte) Krankenschwester bot ihre Dienste unter anderem über eine Internet-Vermittlungsbörse für Pflegekräfte an. Über diese Börse wurde sie von einem ambulanten Pflegedienst für den Einsatz bei einer ganz bestimmten Pflegebedürftigen gebucht, jeweils für einen Zeitraum von maximal zwei Wochen. Auch hier wur-

\title{
FRAGE
}

\section{Fristlose Kündigung wegen einer Bagatelle?}

Inwieweit ist nach einem Diebstahl geringwertiger Sachen eine Kündigung des Arbeitsverhältnisses gerechtfertigt?

In den Medien wird immer wieder von Fällen berichtet, in denen Arbeitgeber scheinbar kleine und sogar nur angebliche Verfehlungen ihrer Mitarbeiter zum Anlass für eine fristlose Kündigung nehmen. Besonders hohe Wellen schlug der Fall einer Berliner Kassiererin, die verdächtigt wurde, zwei von Kunden verlorene Leergutbons im Wert von 1,30 Euro an sich genommen und eingelöst zu haben. Die Kassiererin wurde fristlos gekündigt und verlor ihre Kündigungsschutzklage in erster und zweiter Instanz. Erst in dritter Instanz beim Bundesarbeitsgericht wurde ihre Kündigung für unwirksam erklärt (Emmily-Fall, Urteil vom 10. Juni 2010, Az: 2 AZR 541/09). Das BAG begründete seine Entscheidung damit, dass die Wegnahme der Leergutbons zwar an sich als Grund für eine fristlose Kündigung nach § 626 BGB geeignet sei. In einem zweiten Prüfungsschritt sei jedoch immer eine umfassende Interessenabwägung vorzunehmen. Wegen der langen Dauer von über 30 Jahren, in der das Arbeitsverhältnis unbeanstandet bestanden habe, ging im Emmily-Fall die Interessenabwägung zu Gunsten der Kassiererin aus.

In einem Fall aus dem Jahr 2015 wurde eine Hamburger Krankenschwester, die in der Zentralen Notaufnahme eines Krankenhauses arbeitete, fristlos gekündigt, weil sie für ihre Schicht und sich aus einem Kühlschrank im Pausenraum acht belegte Brötchenhälften genommen hatte, die für externe Rettungssanitäter gedacht waren. Mit ihrer Kündigungsschutzklage beim Arbeitsgericht Hamburg hatte die Krankenschwester Erfolg (Urteil vom 01. Juli 2015, Az.: 27 Ca 87/15). Die Wegnahme der Brötchenhälften stellt, so das Gericht, an sich einen Grund für eine fristlose Kündigung dar. Die Interessenabwägung fiel jedoch auch in diesem Fall zu Gunsten der Arbeitnehmerin aus. Folgende Gründe sprachen für sie: Lange Dauer eines beanstandungsfreien Arbeitsverhältnisses (23 Jahre), Unterhaltspflichten gegenüber zwei Kindern als alleinerziehende Mutter, sofortiges Zugeben des Fehlers, kein heimliches Handeln, kein ausschließlich eigennütziges Handeln wegen der Weitergabe der Brötchen an die Schicht sowie fehlende Wiederholungsgefahr.

Martina Weber

Volljuristin (Ass. jur.)

de die Selbstständigkeit anerkannt (Sozialgericht Hamburg, Urteil vom 27.1.2014, Az. Az. S 10 R 971/08).

\section{Status prüfen lassen}

Letztlich bleibt aufgrund der Einzelfallentscheidungen der Gerichte eine gewisse Unsicherheit bestehen. Dazu abschließend noch ein Tipp: Mitarbeiter können ihren Status bei der Deutschen Rentenversicherung Bund nach $\S 7$ a SGB IV feststellen lassen. Der Vorteil ist Rechtssicherheit, der Nachteil eine möglicherweise negative Entscheidung. Allerdings kann man dagegen immer ein Rechtsmittel einlegen. Zunächst ist ein Widerspruch möglich. Hierzu unbedingt die Frist von einem Monat beachten. Die Behörde muss die Entscheidung nun prüfen und kann sie korrigieren. Macht sie das nicht, so erlässt sie einen Widerspruchsbescheid. Jetzt kann eine Klage helfen. Auch hierzu hat man eine Frist von einem Monat. Wichtig: Unbedingt am Ball bleiben, Hartnäckigkeit hat schon häufig gesiegt!

Eine Überprüfung des Status kann sich aber auch noch auf ganz anderem Weg ergeben: Wenn es zu einem Konflikt kommt, z.B. weil das Krankenhaus oder das Pflegeunternehmen dem Mitarbeiter kündigt, dann kann der Gekündigte beim Arbeitsgericht eine Kündigungsschutzklage erheben. Das Gericht wird gleich zu Beginn prüfen, ob der Mitarbeiter überhaupt als Arbeitnehmer gilt (denn nur dann ist die Klage zulässig). Bescheinigt das Gericht diesen Status, dann hat der Mitarbeiter sein Ziel erreicht und der Kündigungsschutz greift. Allerdings greifen dann auch alle weiteren Folgen, vor allem die Nachzahlungen von Sozialversicherungsabgaben. Das ist für den Arbeitnehmer allerdings weniger ein Problem, weit mehr für den Arbeitgeber.

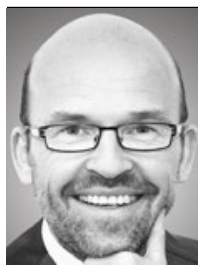

Thorsten Siefarth Rechtsanwalt Sophienstraße 1 80333 München kanzlei@ra-siefarth.de 